\title{
PERIODIC SOLUTIONS OF LINEAR, RICCATI, AND ABEL DYNAMIC EQUATIONS
}

\author{
MARTIN BOHNER, ARMENGOL GASULL, AND CLAUDIA VALLS
}

\begin{abstract}
We study the number of periodic solutions of linear, Riccati and Abel dynamic equations in the time scales setting. In this way, we recover known results for corresponding differential equations and obtain new results for associated difference equations. In particular, we prove that there is no upper bound for the number of isolated periodic solutions of Abel difference equations. One of the main tools introduced to get our results is a suitable Melnikov function. This is the first time that Melnikov functions are used for dynamic equations on time scales.
\end{abstract}

\section{INTRODUCTION AND MAIN RESULTS}

Consider the polynomial periodic differential equations

$$
\frac{\mathrm{d} x}{\mathrm{~d} t}=x^{\prime}=a_{0}(t)+a_{1}(t) x+\cdots+a_{n-1}(t) x^{n-1}+a_{n}(t) x^{n},
$$

where $x, t \in \mathbb{R}$ and $a_{0}, a_{1}, \ldots, a_{n}: \mathbb{R} \rightarrow \mathbb{R}$ are smooth $\omega$-periodic functions. The question of studying its number of $\omega$-periodic solutions in terms of $n$ was proposed by N. G. Lloyd (see [19]) and C. Pugh (see [18]). Notice that (1.1) with $n=1$ (resp. $n=2$ ) is a linear equation (resp. a Riccati equation), and it is well known that linear (resp. Riccati) equations have either a continuum of periodic solutions or at most 1 (resp. 2) periodic solutions, see for instance $[18,20]$. When $n=3,(1.1)$ is called Abel equation, and the more relevant result on it was proved in [18]: For any $k$, there exist equations of the form (1.1), with $a_{i}$ trigonometric $\omega$-periodic polynomials, having at least $k$ isolated $\omega$-periodic solutions. A similar result holds for $n>3$. The results for $n=3$ have interest by themselves, but also because Abel equations appear when studying the number of limit cycles of planar polynomial differential equations, and so, are related to the celebrated Hilbert sixteenth problem, see, for instance, [7, 9-11,13, 14, 16, 18, 21, 24].

In the discrete context, the analogues of the above linear and Riccati periodic difference equations are

$$
x_{n+1}=A_{n}+B_{n} x_{n} \quad \text { and } \quad x_{n+1}=\frac{A_{n}+B_{n} x_{n}}{C_{n}+D_{n} x_{n}}, \quad n \in \mathbb{N},
$$

respectively, where $A_{n}, B_{n}, C_{n}, D_{n}$ are $\omega$-periodic sequences and $\omega \in \mathbb{N}$. As far as we know, there are no agreed-upon versions for difference Abel equations, although some proposals are given in $[4,23]$.

2010 Mathematics Subject Classification. Primary 34N05, Secondary 34C25, 39A06, 39A23.

Key words and phrases. Linear, Riccati, and Abel differential and difference equations, times scales, periodic function, Melnikov function. 
Next, in the time-scales setting, we introduce several dynamic equations that include the known differential or difference equations of linear, Riccati, and Abel differential equations. As usual, these dynamic equations are given using the well-known operators introduced in this theory that can be found for instance in the books $[2,3]$ and that we recall in the Appendix for the sake of completeness. We start with two different forms corresponding to linear dynamic equations. They are

$$
\begin{aligned}
& x^{\Delta}=a(t)+b(t) x, \\
& x^{\Delta}=a(t)-b(t) x^{\sigma},
\end{aligned}
$$

where $a, b \in \mathrm{C}_{\mathrm{rd}}(\mathbb{T}, \mathbb{R})$ are $\omega$-periodic on an $\omega$-periodic time scale $\mathbb{T}$, see the Appendix for the definitions of this space and the notations. We remark that the minus sign in the second equation is only for convenience, to get a simpler expression of the solution of this equation. Notice that, when $\mathbb{T}=\mathbb{R}$, both equations correspond to (1.1) with $n=1$. Similarly, when $\mathbb{T}=\mathbb{N}_{0}$, they correspond to the left-hand equation in (1.2).

For the Riccati case, the corresponding dynamic equations that we consider are

$$
\begin{aligned}
& x^{\Delta}=a(t)+b(t) x+c(t) x x^{\sigma}, \\
& x^{\Delta}=a(t)-b(t) x^{\sigma}+c(t) x x^{\sigma}, \\
& x^{\Delta}=a(t)-b(t) x^{\sigma}-c(t) \frac{x^{2}}{1+\mu(t) c(t) x},
\end{aligned}
$$

where $a, b, c \in \mathrm{C}_{\mathrm{rd}}(\mathbb{T}, \mathbb{R})$ are $\omega$-periodic on an $\omega$-periodic time scale $\mathbb{T}$ and $c \neq 0$. Like in the linear case, when $\mathbb{T}=\mathbb{R}$, all the equations correspond to (1.1) with $n=2$, and, when $\mathbb{T}=\mathbb{N}_{0}$, they correspond to the right-hand equation in (1.2) for certain $A_{n}, B_{n}, C_{n}$ and $D_{n}$ obtained from $a(t), b(t)$, and $c(t)$. For instance, for $(1.5)$ and $\mathbb{T}=\mathbb{R}, x^{\Delta}=x^{\prime}$ and $x x^{\sigma}=x^{2}$. For $\mathbb{T}=\mathbb{N}_{0}, t=n$ and $z(m)=z_{m}, x^{\Delta}(n)=x_{n+1}-x_{n}, x^{\sigma}(n)=x_{n+1}$, and so, $x_{n+1}-x_{n}=a_{n}+b_{n} x_{n}+c_{n} x_{n} x_{n+1}$. In consequence,

$$
x_{n+1}=\frac{a_{n}+\left(1+b_{n}\right) x_{n}}{1-c_{n} x_{n}},
$$

which clearly is a Riccati difference equation.

Finally, the Abel dynamic equations that we will study are

$$
\begin{aligned}
& x^{\Delta}=a(t)+b(t) x+c(t) x x^{\sigma}+d(t) x^{2} x^{\sigma}, \\
& x^{\Delta}=a(t)-b(t) x^{\sigma}+c(t) x x^{\sigma}+d(t) x^{2} x^{\sigma},
\end{aligned}
$$

where $a, b, c, d \in \mathrm{C}_{\mathrm{rd}}(\mathbb{T}, \mathbb{R})$ are $\omega$-periodic on an $\omega$-periodic time scale $\mathbb{T}$ and $d \neq 0$.

If an initial value problem consisting of a dynamic equation and the initial condition $x(0)=\varrho$ has a unique solution, then we denote this solution by $x(\cdot, \varrho)$. In this situation, we can define the Poincaré map as $\Pi(\varrho):=x(\omega, \varrho)-\varrho$. Its fixed points give the initial values of the $\omega$-periodic solutions of the equation. From now on, when we refer to a periodic solution, we always mean $\omega$-periodic solutions.

Our main result is the following.

Theorem 1.1. (i) Consider any of the linear dynamic equations (1.3) or (1.4). If $b$ is regressive, then it has either no periodic solution, or one periodic solutions, or a continuum of periodic solutions. 
(ii) Consider any of the Riccati dynamic equations (1.5), (1.6), or (1.7) with $\mathbb{T} \in$ $\left\{\mathbb{R}, \mathbb{Z}, \mathbb{N}_{0}\right\}$. Then it has either no periodic solution, one or two periodic solutions, or a continuum of periodic solutions.

(iii) Consider the Abel dynamic equations (1.8) or (1.9).

(a) If $\mathbb{T}=\mathbb{R}$, then, for any $k \in \mathbb{N}$, there exists an equation of any of the two given forms with at least $k$ periodic solutions.

(b) If $\mathbb{T}=\mathbb{N}_{0}$ and $\omega \in \mathbb{N}$, then there exists an equation of any of the two given forms with at least $\omega-1$ periodic solutions.

Result (i) when $\mathbb{T} \in\left\{\mathbb{R}, \mathbb{N}_{0}\right\}$ and result (ii) when $\mathbb{T}=\mathbb{R}$ are well known. Result (ii) when $\mathbb{T}=\mathbb{N}_{0}$ is a folklore result. As we will see, for other periodic time scales, it also holds. All these results are proved in Sections 2 and 3. In these sections, we include several examples exhibiting different numbers of periodic solutions for different periodic time scales. We also discuss the problem of determining which of the situations displayed in the theorem holds. As we will see, while in the case $\mathbb{T}=\mathbb{N}_{0}$ this problem can be easily solved, the same question when $\mathbb{T}=\mathbb{R}$ is very difficult, unless a particular periodic solution of the Riccati equation is known. Result (iii) is only known when $\mathbb{T}=\mathbb{R}$. It is new when $\mathbb{T}=\mathbb{N}_{0}$. One of the most important contributions of this work is item (iii) when $\mathbb{T}=\mathbb{N}_{0}$. As a consequence, notice that in the Abel cases to have sharp upper bounds for the number of isolated periodic solutions, some extra hypotheses on the equation must be added. For instance, it is known that for Abel differential equations $\left(n=3\right.$ in (1.1)), the equation has at most 3 of such solutions when $a_{3} \neq 0$ does not change its sign, see $[13,18,22]$. Similar types of results could be addressed for $\mathbb{T}=\mathbb{N}_{0}$.

To prove item (iii), we introduce an analogue of the Melnikov function in the time scales setting. This is the first time that this tool is used in this context. We prove the following result.

Theorem 1.2. Consider the Abel dynamic equation

$$
x^{\Delta}=c(t) x x^{\sigma}+\varepsilon d(t) x^{2} x^{\sigma},
$$

where $c, d \in \mathrm{C}_{\mathrm{rd}}(\mathbb{T}, \mathbb{R})$ and $\varepsilon \in \mathbb{R}$ is a parameter. Define $\bar{C}:=\max _{s \in[0, \omega] \cap \mathbb{T}} C(s)$, where $C(t):=-\int_{0}^{t} c(\tau) \Delta \tau$, and assume that $C(\omega)=0$. For any $\varrho>\bar{C}$, we define the Melnikov function

$$
M(\varrho):=\int_{0}^{\omega} \frac{d(\tau)}{\varrho-C(\tau)} \Delta \tau .
$$

Then, for $|\varepsilon|$ small enough, each simple zero in $(\bar{C}, \infty)$ of $M(\varrho)$ gives an isolated periodic solution of (1.10). This solution tends, when $\varepsilon$ tends to zero, to the periodic solution of (1.10) for $\varepsilon=0$, such that $x(0)=1 / \varrho$.

All our results about Abel dynamic equations are given in Section 4.

\section{LINEAR DYNAMIC EQUATIONS}

Item (i) of Theorem 1.1 is a consequence of the following theorem. Note that it also clarifies which of the three situations given in Theorem 1.1 happens.

Theorem 2.1. Consider the linear dynamic equations (1.3) or (1.4) and assume that $b \in \mathcal{R}$. Then it has either no periodic solutions, or one periodic solutions, or a continuum 
of periodic solutions. Moreover, the initial conditions of the periodic solutions coincide with the solutions of $A+B \varrho=\varrho$, where

$$
\begin{aligned}
& A=\int_{0}^{\omega} e_{b}(\omega, \sigma(\tau)) a(\tau) \Delta \tau, \quad B=e_{b}(\omega, 0) \quad \text { for } \quad(1.3), \quad \text { and } \\
& A=\int_{0}^{\omega} e_{\ominus b}(\omega, \tau) a(\tau) \Delta \tau, \quad B=e_{\ominus b}(\omega, 0) \quad \text { for } \quad(1.4),
\end{aligned}
$$

where the above expressions are introduced in the Appendix. Furthermore, if they have an isolated periodic solution $(B \neq 1)$, then it is

$$
\begin{aligned}
& x(t)=\frac{e_{b}(\omega, 0)}{1-e_{b}(\omega, 0)} \int_{t}^{\omega+t} e_{b}(t, \sigma(\tau)) a(\tau) \Delta \tau \quad \text { for } \quad(1.3), \quad \text { and } \\
& x(t)=\frac{e_{\ominus b}(\omega, 0)}{1-e_{\ominus b}(\omega, 0)} \int_{t}^{\omega+t} e_{\ominus b}(t, \tau) a(\tau) \Delta \tau \quad \text { for } \quad \text { (1.4). }
\end{aligned}
$$

Remark 2.2. (i) Notice that when $\mathbb{T}=\mathbb{R}$, the hypothesis that $b \in \mathcal{R}$ always holds because the graininess is $\mu=0$ and $1+\mu(t) b(t)=1 \neq 0$, see the Appendix.

(ii) When $\mathbb{T}=\mathbb{N}_{0}$, even when $b \notin \mathcal{R}$, the number of the periodic solutions for the linear dynamic equations (1.3) or (1.4) is also given by Theorem 2.1. Let us prove this fact, for instance, for (1.3). By linearity, all its solution can be written as $x(t)=x_{\mathrm{par}}(t)+y(t)$, where $y$ are all the solutions of

$$
y^{\Delta}=b(t) y \Longrightarrow y(n+1)=(1+b(n)) y(n),
$$

and $x_{\text {par }}$ is a particular fixed solution of (1.3). Since $b \notin \mathcal{R}$, for some $n=m$, $1+b(m)=0$, and so, since

$$
y(n)=\prod_{i=0}^{n-1}(1+b(i)),
$$

it holds that for $n \geq m+1, y(n)=0$. Hence all the solutions of (1.3) for $n \geq m+1$ coincide with $x_{\text {par }}$. Then, either the dynamic equation has no periodic solution, or all of them are eventually periodic, but in any case, both situations are covered by the three possibilities given in Theorem 2.1.

Proof of Theorem 2.1. Since $b \in \mathcal{R}$, by using Theorem A.6 of the Appendix, the respective solutions of (1.3) or (1.4), with initial condition $x(0)=\varrho$, are

$$
\begin{aligned}
& x(t)=e_{b}(t, 0) \varrho+\int_{0}^{t} e_{b}(t, \sigma(\tau)) a(\tau) \Delta \tau, \\
& x(t)=e_{\ominus b}(t, 0) \varrho+\int_{0}^{t} e_{\ominus b}(t, \tau) a(\tau) \Delta \tau .
\end{aligned}
$$

The initial conditions that give rise to periodic solutions must be fixed points of the corresponding Poincaré maps. These Poincaré maps are of the form

$$
\Pi(\varrho)=x(\omega)=A+B \varrho .
$$

Hence, these initial conditions are the solutions of $A+B \varrho=\varrho$, as we wanted to prove. Clearly, the equation has no periodic solutions when $B=1, A \neq 0$, and has a continuum 
of them when $B=1$ and $A=0$. Otherwise, there is exactly one periodic solution, and its initial value is $\varrho=A /(1-B)$. For instance, for (1.3), it can be written as

$$
\begin{aligned}
x(t) & =\frac{e_{b}(t, 0) \int_{0}^{\omega} e_{b}(\omega, \sigma(\tau)) a(\tau) \Delta \tau}{1-e_{b}(\omega, 0)}+\int_{0}^{t} e_{b}(t, \sigma(\tau)) a(\tau) \Delta \tau \\
& =\frac{e_{b}(t, 0)}{1-e_{b}(\omega, 0)}\left(\int_{0}^{\omega} e_{b}(\omega, \sigma(\tau)) a(\tau) \Delta \tau+\left(1-e_{b}(\omega, 0)\right) \int_{0}^{t} e_{b}(0, \sigma(\tau)) a(\tau) \Delta \tau\right) \\
& =\frac{e_{b}(t, 0)}{1-e_{b}(\omega, 0)}\left(\int_{t}^{\omega} e_{b}(\omega, \sigma(\tau)) a(\tau) \Delta \tau+\int_{0}^{t} e_{b}(0, \sigma(\tau)) a(\tau) \Delta \tau\right) \\
& =\frac{e_{b}(t, 0)}{1-e_{b}(\omega, 0)}\left(\int_{t}^{\omega} e_{b}(\omega, \sigma(\tau)) a(\tau) \Delta \tau+\int_{\omega}^{\omega+t} e_{b}(\omega, \sigma(\tau)) a(\tau) \Delta \tau\right) \\
& =\frac{e_{b}(t, 0)}{1-e_{b}(\omega, 0)} \int_{t}^{\omega+t} e_{b}(\omega, \sigma(\tau)) a(\tau) \Delta \tau=\frac{e_{b}(\omega, 0)}{1-e_{b}(\omega, 0)} \int_{t}^{\omega+t} e_{b}(t, \sigma(\tau)) a(\tau) \Delta \tau,
\end{aligned}
$$

as we wanted to prove. Notice that we have used several times the semigroup property $e_{b}(t, r) e_{b}(r, s)=e_{b}(t, s)$ given in Theorem A.4 and also, using the periodicity of $a$ and $b$, that $e_{b}(t, r)=e_{b}(t+\omega, r+\omega)$ and

$$
\begin{aligned}
\int_{0}^{t} e_{b}(0, \sigma(\tau)) a(\tau) \Delta \tau & =\int_{\omega}^{\omega+t} e_{b}(0, \sigma(u-\omega)) a(u-\omega) \Delta u \\
& =\int_{\omega}^{\omega+t} e_{b}(0, \sigma(u)-\omega) a(u) \Delta u=\int_{\omega}^{\omega+t} e_{b}(\omega, \sigma(u)) a(u) \Delta u
\end{aligned}
$$

see also [1], in particular, [1, Theorem 2.1 and its proof]. The proof for (1.4) follows similar steps, and we omit it.

2.1. Examples of linear dynamic equations. Now we provide some examples of linear dynamic equations (1.3) for various periodic time scales. Similar examples could be given for (1.4).

Example 2.3. Let $\mathbb{T}=\mathbb{R}$ and $\omega=2 \pi$. First, let $b(t)=\sin (t)$. Then,

$$
B=e_{b}(\omega, 0)=\exp \left(\int_{0}^{2 \pi} \sin (t) \mathrm{d} t\right)=1 .
$$

Let $a=0$. Then, $A=0$ and $\Pi(\varrho) \equiv \varrho$, and by Theorem 2.1, all solutions of $x^{\prime}=x \sin (t)$ are periodic. In fact,

$$
x(t)=\varrho e^{1-\cos (t)} .
$$

Now, let $a=1$. Then, $A=\frac{1}{e} \int_{0}^{2 \pi} e^{\cos (\tau)} \mathrm{d} \tau \neq 0$. Then, $\Pi(\varrho) \equiv \varrho+A$ and the equation has no periodic solution. In fact, the solutions of $x^{\prime}=1+x \sin (t)$ are

$$
x(t)=\varrho e^{1-\cos (t)}+e^{-\cos (t)} \int_{0}^{t} e^{\cos (\tau)} \mathrm{d} \tau .
$$

Finally, let $b=-1$. Then, $B=e^{-2 \pi} \neq 1$. Let $a(t)=2 \sin (t)$. Then, $A=e^{-2 \pi}-1$ and $\Pi(\varrho) \equiv e^{-2 \pi}-1+e^{-2 \pi} \varrho$. Hence, the equation has only one periodic solution with initial condition $\varrho=-1$. In this case, the solutions of $x^{\prime}=2 \sin (t)-x$ are

$$
x(t)=\sin (t)-\cos (t)+(1+\varrho) e^{-t},
$$


and clearly, the only periodic one corresponds to $\varrho=-1$. Note also that, following Theorem 2.1, the periodic solution is

$$
\frac{e^{-2 \pi}}{1-e^{-2 \pi}} \int_{t}^{2 \pi+t} e^{\tau-t} 2 \sin (\tau) \mathrm{d} \tau=\sin (t)-\cos (t) .
$$

Example 2.4. Let $\mathbb{T}=\mathbb{N}_{0}$ and $\omega=4$. First, let $b$ be $\omega$-periodic with

$$
b(0)=-\frac{2}{3}, \quad b(1)=-\frac{1}{2}, \quad b(2)=1, \quad b(3)=2 .
$$

Then,

$$
B=e_{b}(\omega, 0)=(1+b(0))(1+b(1))(1+b(2))(1+b(3))=1 .
$$

Let $a=0$. Then, $A=0$ and $\Pi(\varrho) \equiv \varrho$, and by Theorem 2.1, all solutions of $x^{\Delta}=x b(t)$ are periodic. In fact, they are

$$
x(0)=\varrho, \quad x(1)=\frac{\varrho}{3}, \quad x(2)=\frac{\varrho}{6}, \quad x(3)=\frac{\varrho}{3}, \quad x(4)=\varrho .
$$

Now, let $a$ be 2 -periodic with $a(0)=0, a(1)=1$. Then,

$$
A=\int_{0}^{\omega} e_{b}(\omega, \sigma(\tau)) a(\tau) \Delta \tau=\sum_{\tau=0}^{3} a(\tau) \prod_{j=\tau+1}^{3}(1+b(j))=7
$$

and $\Pi(\varrho) \equiv 7+\varrho$, and the equation has no periodic solution. In fact, the solutions of $x^{\Delta}=a(t)+x b(t)$ are

$$
x(0)=\varrho, \quad x(1)=\frac{\varrho}{3}, \quad x(2)=1+\frac{\varrho}{6}, \quad x(3)=2+\frac{\varrho}{3}, \quad x(4)=7+\varrho .
$$

Finally, let $b$ be $\omega$-periodic with

$$
b(0)=0, \quad b(1)=-\frac{1}{2}, \quad b(2)=-\frac{2}{3}, \quad b(3)=-\frac{3}{4} .
$$

Then,

$$
B=1 \cdot \frac{1}{2} \cdot \frac{1}{3} \cdot \frac{1}{4}=\frac{1}{24} \neq 1 \text {. }
$$

Let $a(t)=23(1+\sin (\pi t))$, i.e., $a$ is $\omega$-periodic with $a(0)=23, a(1)=0, a(2)=23$, $a(3)=46$. In this case, $A=1265 / 24$, and thus $\Pi(\varrho) \equiv 1265 / 24+\varrho / 24$. Solving the equation $\Pi(\varrho)=\varrho$, we get that the unique periodic solution corresponds to the initial condition $\varrho=55$. Indeed, the solutions of $x^{\Delta}=a(t)+x b(t)$ are

$$
x(0)=\varrho, \quad x(1)=23+\varrho, \quad x(2)=\frac{23}{2}+\frac{\varrho}{2}, \quad x(3)=\frac{161}{6}+\frac{\varrho}{6}, \quad x(4)=\frac{1265}{24}+\frac{\varrho}{24} .
$$

Note also that, from the expression of the periodic solution given in Theorem 2.1, the initial condition of the periodic solution is

$$
\varrho=\frac{e_{b}(4,0)}{1-e_{b}(4,0)} \int_{0}^{4} e_{b}(t, \sigma(\tau)) a(\tau) \Delta \tau=\frac{1}{23} \sum_{\tau=0}^{3} a(\tau) e_{b}(0, \tau+1)=1+0+6+48=55 .
$$

In the next two examples, the time scales are not the "usual" ones, but $b \in \mathcal{R}$. 
Example 2.5. Let $\mathbb{T}=\bigcup_{n=0}^{\infty}[2 n, 2 n+1]$ and $\omega=2$. First, let $b$ be $\omega$-periodic with $b(t)=1 /(t+1)$ for $0 \leq t<1$ and $b(1)=-1 / 2$. Then,

$$
B=e_{b}(\omega, 0)=e_{b}(2,1) e_{b}(1,0)=(1+b(1)) \exp \left(\int_{0}^{1} \frac{\mathrm{d} t}{t+1}\right)=1 .
$$

Let $a=0$. Hence, $A=0$ and $\Pi(\varrho) \equiv \varrho$, and all solutions of $x^{\Delta}=x b(t)$ are $\omega$-periodic. They are

$$
x(t)=(t+1) \varrho \quad \text { for } \quad 0 \leq t \leq 1 \quad \text { and } \quad x(2)=(1+b(1)) x(1)=\varrho .
$$

Now, let $a$ be $\omega$-periodic with $a(t)=t+1$ for $0 \leq t \leq 1$. Then, $x^{\Delta}=a(t)+x b(t)$ has no $\omega$-periodic solutions, as the solutions are

$x(t)=(t+1)(t+\varrho) \quad$ for $\quad 0 \leq t \leq 1 \quad$ and $\quad x(2)=a(1)+(1+b(1)) x(1)=\varrho+3=\Pi(\varrho)$.

Finally, let $b$ be $\omega$-periodic with $b(t)=1 /(t+1)$ for $0 \leq t \leq 1$. Then,

$$
B=e_{b}(\omega, 0)=(1+b(1)) \exp \left(\int_{0}^{1} \frac{\mathrm{d} t}{t+1}\right)=3 \neq 1 .
$$

Let $a$ be $\omega$-periodic with $a(t)=t+1$ for $0 \leq t<1, a(1)=3$. The solutions of $x^{\Delta}=a(t)+x b(t)$ are

$x(t)=(t+1)(t+\varrho) \quad$ for $\quad 0 \leq t \leq 1 \quad$ and $\quad x(2)=a(1)+(1+b(1)) x(1)=3 \varrho+6=\Pi(\varrho)$.

Hence, $x$ is $\omega$-periodic if and only if $\varrho=-3$. Note also that from the expression of the periodic orbit in Theorem 2.1, we obtain again that its initial value is

$$
\varrho=-\frac{3}{2}\left\{\int_{0}^{1} e_{b}(0, \tau) a(\tau) \mathrm{d} \tau+e_{b}(0,2) a(1)\right\}=-\frac{3}{2}\left(1+\frac{1}{3} \cdot 3\right)=-3 .
$$

Example 2.6. Let $\mathbb{T}$ be an arbitrary $\omega$-periodic time scale with $0 \in \mathbb{T}$. First, let $b=0$. Then, $B=e_{b}(\omega, 0)=1$. Let $a=0$. Hence, $A=0$ and $\Pi(\varrho) \equiv \varrho$, and all solutions are $\omega$-periodic. In fact, all $x(t) \equiv \varrho$ are constant and hence $\omega$-periodic. Now, let $a=1$. Then, $B=\omega$ and $\Pi(\varrho) \equiv \varrho+\omega$, and the equation has no periodic solution. In fact,

$$
x(t)=t+\varrho .
$$

Finally, let $b$ be $\omega$-periodic with $e_{b}(\omega, 0) \neq 1$ and $a=b$. Then, $A=e_{b}(\omega, 0)-1$ and $B=e_{b}(\omega, 0)$. Hence, $\Pi(\varrho) \equiv e_{b}(\omega, 0)-1+e_{b}(\omega, 0) \varrho$, and the unique periodic solutions starts at $\varrho=-1$. In fact, the solutions of $x^{\Delta}=(1+x) b(t)$ are $x(t)=(\varrho+1) e_{b}(t, 0)-1$, and due to

$$
e_{b}(t+\omega, 0)=e_{b}(t+\omega, t) e_{b}(t, 0)=e_{b}(\omega, 0) e_{b}(t, 0) \neq e_{b}(t, 0)
$$

they are $\omega$-periodic if and only if $\varrho=-1$, and then the solution is $x(t) \equiv-1$.

\section{RicCATI DYNAMiC EQUATiONS}

Instead of proving item (ii) of Theorem 1.1, we state and prove two different stronger results, one for $\mathbb{T}=\mathbb{R}$ and the other one for $\mathbb{T}=\mathbb{N}_{0}$, that clearly imply it. As we will see, these results also give information to know in each case which is the exact the number of periodic solutions of the Riccati equation. In general, the answer is unknown when $\mathbb{T}=\mathbb{R}$, unless a particular periodic solutions is known, see [8] or Proposition 3.1 below. 
On the other hand, when $\mathbb{T}=\mathbb{N}_{0}$, the question can be totally solved, see Proposition 3.2 .

Proposition 3.1. Let

$$
x^{\prime}=a(t)+b(t) x+c(t) x^{2}
$$

be an $\omega$-periodic Riccati differential equation and let $x=x_{0}(t)$ be an explicit $\omega$-periodic solution of (3.1). Then, there exist four computable real numbers, $A, B, C, D$ such that if we denote by $\mathcal{S}$ the set of all real solutions @ of the equation

$$
\frac{A+B \varrho}{C+D \varrho}=\varrho,
$$

then the periodic solutions of (3.1) are given by the solutions of (3.1) with initial value $x(0)=\varrho \in \mathcal{S}$ that are well defined for all $t \in[0, \omega]$.

Proof. With the change of variables $y=1 /\left(x-x_{0}\right)$, we can write (3.1) as the linear differential equation

$$
y^{\prime}=-c(t)-\left(2 c(t) x_{0}(t)+b(t)\right) y
$$

Solving it and going back to the variable $x$, we get that the solution of (3.1) satisfying $x(0)=\varrho$ can be written as

$$
x(t)=\frac{\mathcal{A}(t)+\mathcal{B}(t) \varrho}{\mathcal{C}(t)+\mathcal{D}(t) \varrho}
$$

for some functions $\mathcal{A}, \mathcal{B}, \mathcal{C}, \mathcal{D}$ that depend on $a, b, c$, and $x_{0}$, but not on $\varrho$. Therefore, the Poincaré map is the Möbius transformation

$$
\Pi(\varrho)=\frac{\mathcal{A}(\omega)+\mathcal{B}(\omega) \varrho}{\mathcal{C}(\omega)+\mathcal{D}(\omega) \varrho} .
$$

The periodic solutions satisfy $\Pi(\varrho)=\varrho$. Hence, we obtain that the initial conditions of the periodic solutions must satisfy (3.2). The solutions starting at some of these values, and defined for all $t \in[0, \omega]$, will give the actual periodic solutions of the differential equation.

Proposition 3.2. Let

$$
x_{n+1}=\frac{A_{n}+B_{n} x_{n}}{C_{n}+D_{n} x_{n}}=f_{n}\left(x_{n}\right)
$$

be an w-periodic Riccati difference equation. Define

and

$$
U_{i}:=\left(\begin{array}{cc}
B_{i} & A_{i} \\
D_{i} & C_{i}
\end{array}\right), \quad i=0, \ldots, \omega-1
$$

$$
U:=U_{\omega-1} U_{\omega-2} \cdots U_{1} U_{0}=\left(\begin{array}{cc}
B & A \\
D & C
\end{array}\right) .
$$

Denote by $\mathcal{S}$ the set of all real solutions $\varrho$ of the equation

$$
\frac{A+B \varrho}{C+D \varrho}=\varrho
$$

Then, the periodic solutions of (3.3) are given by the solutions of (3.3) with initial value $x(0)=\varrho \in \mathcal{S}$ that are well defined for all $i=1,2, \ldots, \omega$. 
Proof. Given a $2 \times 2$ matrix

$$
V=\left(\begin{array}{ll}
b & a \\
d & c
\end{array}\right), \quad \text { we define } \quad \tau_{V}(x):=\frac{b x+a}{d x+c} .
$$

With this notation, we can rewrite (3.3) as

$$
x_{n+1}=f_{n}\left(x_{n}\right)=\frac{B_{n} x_{n}+A_{n}}{D_{n} x_{n}+C_{n}}=\tau_{U_{n}}\left(x_{n}\right) .
$$

First notice that, for any $i, j \in \mathbb{N}_{0}$,

$$
\left(f_{i} \circ f_{j}\right)(x)=\frac{B_{i} \frac{B_{j} x+A_{j}}{D_{j} x+C_{j}}+A_{i}}{D_{i} \frac{B_{j} x+A_{j}}{D_{j} x+C_{j}}+C_{i}}=\frac{\left(B_{i} B_{j}+A_{i} D_{j}\right) x+\left(B_{i} A_{j}+A_{i} C_{j}\right)}{\left(D_{i} B_{j}+C_{i} D_{j}\right) x+\left(D_{i} A_{j}+C_{i} C_{j}\right)}=\tau_{U_{i} U_{j}}(x) .
$$

The $\omega$-periodic solutions are given by the solutions of $\left(f_{\omega-1} \circ f_{\omega-2} \circ \cdots \circ f_{0}\right)(\varrho)=\varrho$, or equivalently, by the solutions of $\tau_{U_{\omega-1} U_{\omega-2} \cdots U_{1} U_{0}}(\varrho)=\tau_{U}(\varrho)=\varrho \in \mathcal{S}$ for which the corresponding orbits are well defined.

3.1. Examples of Riccati dynamic equations when $\mathbb{T} \in\left\{\mathbb{R}, \mathbb{N}_{0}\right\}$. We present examples only for (1.6). Examples for (1.5) and (1.7) can be constructed similarly.

Example 3.3 (See also [10]). An example of (1.6) with $\mathbb{T}=\mathbb{R}, \omega=2 \pi$, and two $\omega$ periodic solutions is $a(t)=1+\sin (t)+\cos (t)-\cos ^{2}(t), b(t)=1+2 \sin (t)$, and $c=-1$. Its solutions are

$$
x(t)=\sin (t)+\frac{\varrho}{\varrho+(1-\varrho) e^{t}},
$$

and they are $\omega$-periodic if and only if either $\varrho=0$ or $\varrho=1$. These periodic solutions are $\sin (t)$ and $1+\sin (t)$, respectively.

Example 3.4. Examples of (1.6) with $\mathbb{T}=\mathbb{N}_{0}$ and either one periodic solution or no periodic solution are the following. If $\omega=2$ and $a, b, c$ are 2-periodic with

$$
a(0)=1, \quad a(1)=-1, \quad b(0)=1, \quad b(1)=1, \quad c(0)=1, \quad c(1)=-1,
$$

then there is a unique solution of (1.6) satisfying

$$
x(1)=\frac{1+2 \varrho}{2+2 \varrho} \text { and } \quad x(2)=\varrho .
$$

On the other hand, if we let $a, b, c$ be 2-periodic with

$$
a(0)=1, \quad a(1)=0, \quad b(0)=1, \quad b(1)=0, \quad c(0)=1, \quad c(1)=-2,
$$

then the unique solution of (1.6) satisfies

$$
x(1)=\frac{1+2 \varrho}{2+2 \varrho} \text { and } \quad x(2)=-\frac{1+2 \varrho}{2 \varrho}
$$

and is never 2 -periodic as $2 \varrho^{2}+2 \varrho+1$ has no real zero. 
3.2. Riccati equations on other periodic time scales. Although in item (ii) of Theorem 1.1 , we only consider the time scales $\mathbb{T} \in\left\{\mathbb{R}, \mathbb{Z}, \mathbb{N}_{0}\right\}$, the same results holds in a more general setting. Assume for instance that the Riccati dynamic equation (1.6) has an $\omega$-periodic solution $x=x_{0}$ (otherwise, we are done). We introduce the classical change of variable $y=1 /\left(x-x_{0}\right)$. By using that $(1 / z)^{\Delta}=-z^{\Delta} /\left(z z^{\sigma}\right)$ and $y^{\sigma}=y+\mu y^{\Delta}$ (see the Appendix), we obtain that $y$ satisfies

$$
\begin{aligned}
y^{\Delta} & =-\frac{x^{\Delta}-x_{0}^{\Delta}}{\left(x-x_{0}\right)\left(x^{\sigma}-x_{0}^{\sigma}\right)}=\left(x_{0}^{\Delta}-x^{\Delta}\right) y y^{\sigma}=\left(a-b x_{0}^{\sigma}+c x_{0} x_{0}^{\sigma}-a+b x^{\sigma}-c x x^{\sigma}\right) y y^{\sigma} \\
& =b y-c\left(\left(\frac{1}{y}+x_{0}\right)\left(\frac{1}{y^{\sigma}}+x_{0}^{\sigma}\right)-x_{0} x_{0}^{\sigma}\right) y y^{\sigma}=b y-c\left(\frac{1}{y y^{\sigma}}+\frac{x_{0}}{y^{\sigma}}+\frac{x_{0}^{\sigma}}{y}\right) y y^{\sigma} \\
& =b y-c-c x_{0} y-c x_{0}^{\sigma} y^{\sigma}=-\left(c x_{0}+c x_{0}^{\sigma}-b\right) y-c-\mu c x_{0}^{\sigma} y^{\Delta},
\end{aligned}
$$

and so

$$
y^{\Delta}=-\frac{c(t)}{1+\mu(t) c(t) x_{0}(\sigma(t))}-\frac{c(t) x_{0}(t)+c(t) x_{0}(\sigma(t))-b(t)}{1+\mu(t) c(t) x_{0}(\sigma(t))} y .
$$

This new equation, whenever it is well defined, is an $\omega$-periodic linear dynamic equation of the form (1.3). If we are under the hypotheses of item (i) of Theorem 1.1, then it has zero, one, or a continuum of $\omega$-periodic solutions. Undoing the change of variables, we get that our initial dynamic Riccati equation has either no periodic solution, one or two periodic solutions, or a continuum of periodic solutions.

If we start with (1.5) and follow the same steps, we also arrive at a linear dynamic equation, in this case of type (1.4), namely

$$
y^{\Delta}=\frac{c(t)}{1+\mu(t) c(t) x_{0}(t)}-\frac{b(t)-c(t) x_{0}(t)-c(t) x_{0}(\sigma(t))}{1+\mu(t) c(t) x_{0}(t)} y^{\sigma} .
$$

The computations for (1.7) are again similar, and we skip all the details.

We end this section with some examples of Riccati equations (1.7) in a different time scale setting.

Example 3.5. We give two examples with $\mathbb{T}=\bigcup_{n=0}^{\infty}[2 n \pi,(2 n+1) \pi]$ and such that they have either two periodic solutions or exactly one periodic solution. Consider (1.7) with $\omega=2 \pi$. Let $a, b, c$ be $\omega$-periodic with

$$
\begin{gathered}
a(t)=1+\sin (t)+\cos (t)-\cos ^{2}(t) \quad \text { for } \quad 0 \leq t<\pi \quad \text { and } a(\pi)=0, \\
b(t)=1+2 \sin (t) \quad \text { for } \quad 0 \leq t<\pi \quad \text { and } \quad b(\pi)=\frac{1}{1-\pi},
\end{gathered}
$$

$c=-1$. Then, a periodic solution of $(1.7)$ is $x_{0}(t)=\sin (t)$. By letting $y=1 /\left(x-x_{0}\right)$, for any other solution $x$ of (1.7), we find that $y^{\prime}=-1+y$ on $[0, \pi]$ and

$$
\frac{y(2 \pi)-y(\pi)}{\pi}=-(1+\pi b(\pi))+b(\pi) y(\pi)=-\frac{1}{1-\pi}+\frac{1}{1-\pi} y(\pi)
$$

(note that $x_{0}(\pi)=0$ ), and the solution satisfies $y(t)=(y(0)-1) e^{t}-1$ for $0 \leq t \leq \pi$ and

$$
y(2 \pi)=-\frac{\pi}{1-\pi}+\frac{1}{1-\pi}\left[(y(0)-1) e^{\pi}+1\right]=1+\frac{1}{1-\pi}(y(0)-1) e^{\pi},
$$


and this equals $y(0)$ if and only if $y(0)=1$. Thus, the unique $\omega$-periodic solution is $y(t) \equiv 1$. Hence, $x_{1}(t)=1+\sin (t)$ is one last periodic solution of (1.7). Thus, (1.7) has exactly two periodic solutions with $x(0)=\varrho \in\{0,1\}$.

Now, let $a, b, c$ be as before except $b(\pi)=\left(e^{-\pi}-1\right) / \pi$. Again, $x_{0}(t)=\sin (t)$ is an $\omega$-periodic solution of (1.7). By assuming that $x$ is another $\omega$-periodic solution of (1.7) and letting $y=1 /\left(x-x_{0}\right)$, we also find that $y^{\prime}=-1+y$ on $[0, \pi]$ and

$$
\frac{y(2 \pi)-y(\pi)}{\pi}=-(1+\pi b(\pi))+b(\pi) y(\pi)=-e^{-\pi}+\frac{e^{-\pi}-1}{\pi} y(\pi)
$$

(note that $x_{0}(\pi)=0$ ), and the solution satisfies $y(t)=(y(0)-1) e^{t}-1$ for $0 \leq t \leq \pi$. Hence, it can be seen that $y(0) \neq y(2 \pi)$. Thus, (1.7) has exactly one periodic solution with $x(0)=\varrho=0$.

\section{Abel Dynamic EQUATions}

This section contains the proofs of the main results of this paper, Theorem 1.2 and item (iii) of Theorem 1.1. We will use the following well-known result about Chebyshev systems, see [15]. See for instance [12] for some examples of Chebyshev systems.

Recall that, given smooth functions $f_{0}, \ldots, f_{n}$ on an open interval $L$, it is said that $\left(f_{0}, \ldots, f_{n}\right)$ is an extended complete Chebyshev system (ECT-system) on $L$ if for all $k=0,2, \ldots, n$, any nontrivial linear combination

$$
\alpha_{0} f_{0}(x)+\ldots+\alpha_{k} f_{k}(x)
$$

with $\alpha_{j} \in \mathbb{R}$ has at most $k$ isolated zeros on $L$ counting multiplicity. We remark that it is easy to see that given a linear combination of $n+1$ functions, as in (4.1) with $k=n$, there always exist $\alpha_{j}$ such that it has at least $n$ zeros in $L$. When the functions form an ECT-system, we can moreover ensure that these $n$ zeros are simple.

Proposition 4.1. $\left(f_{0}, \ldots, f_{n}\right)$ is an ECT-system on $L$ if and only if for all $k=0,1, \ldots n$, $W\left[f_{0}, \ldots, f_{k}\right](x) \neq 0$ for all $x \in L$, where

$$
W\left[f_{0}, \ldots, f_{k}\right](x):=\operatorname{det}\left(f_{j}^{(i)}(x)\right)_{0 \leq i, j \leq k}
$$

is the Wronskian of $\left(f_{0}, \ldots, f_{k}\right)$ at $x \in L$.

Proof of Theorem 1.2. Consider the change of variables $y=1 / x$ in (1.10). We get

$$
y^{\Delta}=-\frac{x^{\Delta}}{x x^{\sigma}}=-\frac{c x x^{\sigma}+\varepsilon d x^{2} x^{\sigma}}{x x^{\sigma}}=-c-\varepsilon \frac{d}{y}, \quad y(0)=\varrho .
$$

We can write $y=\alpha+\varepsilon w+\mathrm{O}\left(\varepsilon^{2}\right)$ with $y(0)=\varrho$, that is, $\alpha(0)=\varrho, w(0)=0$, and so

$$
\alpha^{\Delta}+\varepsilon w^{\Delta}+\mathrm{O}\left(\varepsilon^{2}\right)=-c-\varepsilon \frac{d}{\alpha+\varepsilon w+\mathrm{O}\left(\varepsilon^{2}\right)}=-c-\varepsilon \frac{d}{\alpha}+\mathrm{O}\left(\varepsilon^{2}\right) .
$$

It is clear that

$$
\alpha^{\Delta}=-c(t), \quad \alpha(0)=\varrho .
$$

Solving this equation (see the Appendix), we get

$$
\alpha(t)-\varrho=-\int_{0}^{t} c(\tau) \Delta \tau=C(t)
$$


that is, $\alpha(t)=\varrho+C(t)$. Since $C(\omega)=0$ by assumption, for any $\varrho>\bar{C}$, all solutions $\alpha$ are $\omega$-periodic (use [1, Theorem 2.1]). It follows from (4.3) that $w$ satisfies

$$
w^{\Delta}=-\frac{d(t)}{\alpha(t)}=-\frac{d(t)}{\varrho-C(t)}, \quad w(0)=0 .
$$

Again using the antiderivative, we conclude that

Therefore,

$$
w(t)=w(0)-\int_{0}^{t} \frac{d(\tau)}{\varrho-C(\tau)} \Delta \tau=-\int_{0}^{t} \frac{d(\tau)}{\varrho-C(\tau)} \Delta \tau .
$$

$$
y(t)=\varrho+C(t)-\varepsilon \int_{0}^{t} \frac{d(\tau)}{\varrho-C(\tau)} \Delta \tau+\mathrm{O}\left(\varepsilon^{2}\right) .
$$

The periodic solutions of (4.2) must satisfy $y(\omega)=\varrho$, that is,

$$
\varrho=\varrho+C(\omega)-\varepsilon \int_{0}^{\omega} \frac{d(\tau)}{\varrho-C(\tau)} \Delta \tau+\mathrm{O}\left(\varepsilon^{2}\right)=\varrho-\varepsilon M(\varrho)+\mathrm{O}\left(\varepsilon^{2}\right),
$$

i.e., $D(\varrho, \varepsilon):=-M(\varrho)+\mathrm{O}(\varepsilon)=0$. It follows from the implicit function theorem applied to $D(\varrho, \varepsilon)=0$ that any simple zero of $M(\varrho), \varrho=\varrho^{*} \neq 0$, gives rise, for $|\varepsilon|$ sufficiently small, to an isolated solution of $D(\varrho, \varepsilon)=0$ that tends to $\varrho^{*}$ when $\varepsilon$ tends to zero. This concludes the proof.

Proof of (a) in item (iii) of Theorem 1.1. In view of Theorem 1.2, we can reduce the proof to consider an Abel differential equation of the form (1.10), that is,

$$
x^{\prime}=c(t) x^{2}+\varepsilon d(t) x^{3},
$$

and then find enough simple nonzero solutions of its Melnikov function $M(\varrho)$ given in (1.11), namely

$$
M(\varrho)=\int_{0}^{\omega} \frac{d(\tau)}{\varrho-C(\tau)} \Delta \tau=\int_{0}^{\omega} \frac{d(\tau)}{\varrho-C(\tau)} \mathrm{d} \tau .
$$

Now, following $[11,18]$, we show that for fixed $k \in \mathbb{N}$, there exist functions $C$ and $d$ such that the above Melnikov function has at least $k$ simple zeros in $(\bar{C}, \infty)$. Take $c(t)=2 \pi \cos (2 \pi t / \omega) / \omega$. Then

$$
C(t)=-\frac{2 \pi}{\omega} \int_{0}^{t} \cos \left(\frac{2 \pi s}{\omega}\right) \mathrm{d} s=\sin \left(\frac{2 \pi t}{\omega}\right) \quad \text { and } \quad \bar{C}=\max _{t \in[0, \omega]} C(t)=1 .
$$

Take now $z:=1 / \varrho$, which is defined in a neighborhood of zero of the form $(0,1)$, and define $H_{d}(z):=M(\varrho)$ with this choice of $c$, that is,

$$
H_{d}(z)=z \int_{0}^{\omega} \frac{d(s)}{1-z \sin (2 \pi s / \omega)} \mathrm{d} s=z G_{d}(z)
$$

where

$$
G_{d}(z):=\int_{0}^{\omega} \frac{d(s)}{1-z \sin (2 \pi s / \omega)} \mathrm{d} s .
$$

Fix $k \in \mathbb{N}$ and an arbitrary polynomial $p(z)$ of degree $k$. We will show that there exists $d(t)$ of the form $d(t)=\sum_{j=0}^{k} \beta_{j} d_{j}(t)$, with $d_{j}(t)=\sin ^{j}(2 \pi t / \omega)$ and $\beta_{j} \in \mathbb{R}$, such that

$$
G_{d}(z)=p(z)+\mathrm{O}\left(z^{k+1}\right) .
$$


Indeed, notice that

$$
G_{d_{j}}(z)=\sum_{\ell=0}^{k} \int_{0}^{\omega} \sin ^{j+\ell}\left(\frac{2 \pi s}{\omega}\right) z^{\ell}+\mathrm{O}\left(z^{k+1}\right)=: \sum_{\ell=0}^{k} c_{j, \ell} z^{\ell}+\mathrm{O}\left(z^{k+1}\right) .
$$

Observe also that if we define the $(k+1) \times(k+1)$ matrix $C=\left(c_{j, \ell}\right)_{0 \leq j, \ell \leq k}$, then $\operatorname{det} C \neq 0$ because it can be associated to the inner product $f \cdot g=\int_{0}^{\omega} f(t) g(t) \mathrm{d} t$, with the basis $\left\langle d_{0}, d_{1}, \ldots, d_{k}\right\rangle$. Then, since $G_{d}$ is linear in $d$, given any polynomial $p(z)$, there exist unique values $\beta_{0}, \ldots, \beta_{k}$ such that its associated $d$ satisfies (4.4). Now the fact that $G_{d}(z)$ has $k$ simple zeros in a small enough neighborhood of the origin follows from Proposition 4.1. This is so, because clearly $\left(1, z, \ldots, z^{k}\right)$ is an ECT-system in $L=\mathbb{R}$, and thus the same holds, but in $L=(0, \varepsilon)$, for $\varepsilon>0$ small enough, for the functions $\left.\left(1, z, \ldots, z^{k}+\mathrm{O}\left(z^{k+1}\right)\right)\right)$. Hence, for a suitable choice of $\beta_{j}, j=0, \ldots, k$, the corresponding $G_{d}$ has $k$ simple zeros in $(0, \varepsilon)$.

Proof of (b) in item (iii) of Theorem 1.1. Again, in view of Theorem 1.2, we can reduce its proof to consider an Abel difference equation of the form (1.10), $x^{\Delta}=c x x^{\sigma}+\varepsilon d x^{2} x^{\sigma}$ with $\mathbb{T}=\mathbb{N}_{0}$ and $\omega=k$. We want to find enough simple nonzero solutions of the associated Melnikov function $M(\varrho)$. In this case,

$$
M(\varrho)=\int_{0}^{\omega} \frac{d(\tau)}{\varrho-C(\tau)} \Delta \tau=\sum_{\ell=0}^{k-1} \frac{d_{\ell}}{\varrho-C_{\ell}} .
$$

Consider the set of functions $\mathcal{C}=\left(\left(\varrho-C_{0}\right)^{-1}, \ldots,\left(\varrho-C_{k-1}\right)^{-1}\right)$. It is not difficult to prove that the Wronskians of $\left(\left(\varrho-C_{0}\right)^{-1}, \ldots,\left(\varrho-C_{j}\right)^{-1}\right)$, for $j=0,1, \ldots, k$, do not vanish for $\varrho \in L:=\left(\max _{j=0,1, \ldots, k} C_{j}, \infty\right)$, because these determinants are of Vandermonde type. Hence, by Proposition $4.1, \mathcal{C}$ is an ECT-system on $L$. and, therefore, there exist $d_{\ell}$, $\ell=0, \ldots, k-1$ such that $M(\varrho)$ has $k-1$ simple zeros in $L$, as we wanted to prove.

\section{APPENDIX}

In this appendix, we recall the following concepts related to the notion of time scales. For more details of time scale analysis, we refer the reader to the two books by Bohner and Peterson $[2,3]$. A time scale $\mathbb{T}$ is an arbitrary nonempty closed subset of the real numbers $\mathbb{R}$. The cases when $\mathbb{T}=\mathbb{R}, \mathbb{T}=\mathbb{N}_{0}$, and $\mathbb{T}=\mathbb{Z}$ represent the classical theories of differential and difference calculus. In this paper, we consider only $\omega$-periodic time scales, i.e., we assume that $\omega \in \mathbb{R}$ is such that

$$
t \in \mathbb{T} \text { always implies } t+\omega \in \mathbb{T} \text {. }
$$

See $[1,5,6,17,25]$ for results related to periodic time scales. Moreover, in this paper, all considered time scales $\mathbb{T}$ satisfy $\sup \mathbb{T}=\infty$, and so we define the forward jump operator $\sigma: \mathbb{T} \rightarrow \mathbb{T}$ by

$$
\sigma(t):=\inf \{s \in \mathbb{T}: s>t\} \quad \text { for all } t \in \mathbb{T} .
$$

The graininess $\mu: \mathbb{T} \rightarrow[0, \infty)$ is defined by

$$
\mu(t):=\sigma(t)-t \quad \text { for all } \quad t \in \mathbb{T} .
$$


For any function $f: \mathbb{T} \rightarrow \mathbb{R}$, we write $f^{\sigma}$ for $f \circ \sigma$. For $t \in \mathbb{T}$, we define $f^{\Delta}(t)$ to be the number (if it exists) with the property that given any $\varepsilon>0$, there is a neighborhood $U$ of $t$ with

$$
\left|f(\sigma(t))-f(s)-f^{\Delta}(t)(\sigma(t)-s)\right| \leq \varepsilon|\sigma(t)-s| \quad \text { for all } \quad s \in U .
$$

In this case, we say $f^{\Delta}(t)$ is the (delta) derivative of $f$ at $t$. If $f$ is (delta) differentiable at any $t \in \mathbb{T}$, then $f^{\Delta}: \mathbb{T} \rightarrow \mathbb{R}$ is called the delta derivative of $f$. Next, if $F: \mathbb{T} \rightarrow \mathbb{R}$ is an antiderivative of $f$, i.e., $F^{\Delta}=f$, then the Cauchy delta integral of $f$ is defined by

$$
\int_{a}^{t} f(s) \Delta s:=F(t)-F(a)
$$

where $a \in \mathbb{T}$ is fixed. It is known [2, Theorem 1.74] that so-called $r d$-continuous functions always possess antiderivatives. A function $f: \mathbb{T} \rightarrow \mathbb{R}$ is called rd-continuous if it is continuous in right-dense points (i.e., points $t$ in which $\sigma(t)=t$ ) and the left-hand sided limits exist in left-dense points (i.e., points $t$ in which $\rho(t)=t$, where the backward jump operator $\rho: \mathbb{T} \rightarrow \mathbb{T}$ is defined in a fashion corresponding to the forward jump operator $\sigma)$. The set of all rd-continuous functions $f: \mathbb{T} \rightarrow \mathbb{R}$ is abbreviated by $\mathrm{C}_{\mathrm{rd}}=\mathrm{C}_{\mathrm{rd}}(\mathbb{T})=$ $\mathrm{C}_{\mathrm{rd}}(\mathbb{T}, \mathbb{R})$.

Example A.1. Note that if $\mathbb{T}=\mathbb{R}$, then

$$
\sigma(t)=t, \quad \mu(t)=0, \quad f^{\Delta}=f^{\prime}, \quad \text { and } \quad \int_{a}^{b} f(t) \Delta t=\int_{a}^{b} f(t) \mathrm{d} t
$$

for $a, b \in \mathbb{R}$ with $a<b$. If $\mathbb{T}=\mathbb{Z}$ or $\mathbb{T}=\mathbb{N}_{0}$, then

$$
\sigma(t)=t+1, \quad \mu(t)=1, \quad f^{\Delta}=\Delta f, \quad \text { and } \quad \int_{a}^{b} f(t) \Delta t=\sum_{t=a}^{b-1} f(t)
$$

for $a, b \in \mathbb{Z}$ or $a, b \in \mathbb{N}_{0}$ with $a<b$. If $\mathbb{T}=h \mathbb{Z}$ with $h>0$, then

$$
\sigma(t)=t+h, \quad \mu(t)=h, \quad \text { and } \quad \int_{a}^{b} f(t) \Delta t=h \sum_{k=0}^{\frac{b-a-h}{h}} f(a+k h)
$$

for $a, b \in h \mathbb{Z}$.

Now we collect those known time scales results that will be used frequently throughout this paper. First, the simple useful formula [2, Theorem 1.16]

$$
f^{\sigma}=f+\mu f^{\Delta}
$$

holds whenever $f: \mathbb{T} \rightarrow \mathbb{R}$ is differentiable. The product rule and quotient rule $[2$, Theorem 1.20] for the derivative of the product $f g$ and the quotient $f / g$ (with $g(t) \neq 0$ for all $t \in \mathbb{T}$ ) of two differentiable functions $f, g: \mathbb{T} \rightarrow \mathbb{R}$ read

$$
(f g)^{\Delta}=f^{\Delta} g+f^{\sigma} g^{\Delta}=f g^{\Delta}+f^{\Delta} g^{\sigma} \quad \text { and } \quad\left(\frac{f}{g}\right)^{\Delta}=\frac{f^{\Delta} g-f g^{\Delta}}{g g^{\sigma}} .
$$


Next, we introduce the time scales exponential function. To this end, let $\mathcal{R}=\mathcal{R}(\mathbb{T})=$ $\mathcal{R}(\mathbb{T}, \mathbb{R})$ be the set of all rd-continuous functions $p: \mathbb{T} \rightarrow \mathbb{R}$ that are regressive, i.e., that satisfy

$$
1+\mu(t) p(t) \neq 0 \quad \text { for all } \quad t \in \mathbb{T} \text {. }
$$

Theorem A.2 (See [2, Theorem 2.33]). If $p \in \mathcal{R}$ and $t_{0} \in \mathbb{T}$, then the initial value problem

$$
y^{\Delta}=p(t) y, \quad y\left(t_{0}\right)=1
$$

has a unique solution.

Definition A.3. If $p \in \mathcal{R}$ and $t_{0} \in \mathbb{T}$, then the unique solution of (A.5) is called the exponential function on the time scale and is denoted by $e_{p}\left(\cdot, t_{0}\right)$.

Some useful properties of the dynamic exponential function are the following.

Theorem A.4 (See [2, Theorem 2.36]). If $p \in \mathcal{R}$, then

(1) $e_{0}(t, s)=1$, and $e_{p}(t, t)=1$,

(2) the semigroup property holds: $e_{p}(t, r) e_{p}(r, s)=e_{p}(t, s)$.

Example A.5. In fact, when $\mathbb{T}=\mathbb{R}$ and $\mathbb{T}=\mathbb{Z}$, we have

$$
e_{p}\left(t, t_{0}\right)=e^{\int_{t_{0}}^{t} p(s) \mathrm{d} s} \quad \text { and } \quad e_{p}\left(t, t_{0}\right)=\prod_{s=t_{0}}^{t-1}(1+p(s))
$$

respectively.

Now the two variation of constants results [2, Theorem 2.74 and Theorem 2.77] that are used in this paper read as follows.

Theorem A.6 (Variation of Constants). Suppose $p \in \mathcal{R}$ and $f \in \mathrm{C}_{\mathrm{rd}}$. Let $t_{0} \in \mathbb{T}$ and $x_{0} \in \mathbb{R}$. The unique solution of the IVP

$$
x^{\Delta}=p(t) x+f(t), \quad x\left(t_{0}\right)=x_{0}
$$

is given by

$$
x(t)=e_{p}\left(t, t_{0}\right) x_{0}+\int_{t_{0}}^{t} e_{p}(t, \sigma(s)) f(s) \Delta s .
$$

The unique solution of the IVP

$$
x^{\Delta}=-p(t) x^{\sigma}+f(t), \quad x\left(t_{0}\right)=x_{0}
$$

is given by

$$
x(t)=e_{\ominus p}\left(t, t_{0}\right) x_{0}+\int_{t_{0}}^{t} e_{\ominus p}(t, s) f(s) \Delta s
$$

provided that we introduce the "circle minus" $\ominus$ by

$$
\ominus p=\frac{-p}{1+\mu p}
$$




\section{ACKNOWLEDGMENTS}

The second author is partially supported by the MINECO/FEDER MTM2016-77278$\mathrm{P}$ and AGAUR 2017-SGR-1617 grants. The third author is partially supported by FCT/Portugal through PEst-OE/EEI/LA0009/2013 (CAMGSD).

\section{REFERENCES}

[1] Li Bi, Martin Bohner, and Meng Fan. Periodic solutions of functional dynamic equations with infinite delay. Nonlinear Anal., 68(5):1226-1245, 2008.

[2] Martin Bohner and Allan Peterson. Dynamic equations on time scales. Birkhäuser Boston, Inc., Boston, MA, 2001. An introduction with applications.

[3] Martin Bohner and Allan Peterson. Advances in dynamic equations on time scales. Birkhäuser Boston, Inc., Boston, MA, 2003.

[4] Martin Bohner and Sabrina H. Streipert. Abel dynamic equations of first and second kind. Georgian Math. J., 22(3):341-348, 2015.

[5] Martin Bohner and Sabrina H. Streipert. The Beverton-Holt equation with periodic growth rate. Int. J. Math. Comput., 26(4):1-10, 2015.

[6] Martin Bohner and Howard Warth. The Beverton-Holt dynamic equation. Appl. Anal., 86(8):10071015, 2007.

[7] Marc Carbonell and Jaume Llibre. Limit cycles of a class of polynomial systems. Proc. Roy. Soc. Edinburgh Sect. A, 109(1-2):187-199, 1988.

[8] Anna Cima, Armengol Gasull, and Víctor Mañosa. Dynamics of some rational discrete dynamical systems via invariants. Internat. J. Bifur. Chaos Appl. Sci. Engrg., 16(3):631-645, 2006.

[9] James Devlin, Noel G. Lloyd, and Jane M. Pearson. Cubic systems and Abel equations. J. Differential Equations, 147(2):435-454, 1998.

[10] Armengol Gasull. From Abel's differential equations to Hilbert's sixteenth problem. Butl. Soc. Catalana Mat., 28(2):123-146, 233, 2013.

[11] Armengol Gasull and Antoni Guillamon. Limit cycles for generalized Abel equations. Internat. J. Bifur. Chaos Appl. Sci. Engrg., 16(12):3737-3745, 2006.

[12] Armengol Gasull, J. Tomás Lázaro, and Joan Torregrosa. On the Chebyshev property for a new family of functions. J. Math. Anal. Appl., 387(2):631-644, 2012.

[13] Armengol Gasull and Jaume Llibre. Limit cycles for a class of Abel equations. SIAM J. Math. Anal., 21(5):1235-1244, 1990.

[14] Yulij S. Ilyashenko. Centennial history of Hilbert's 16th problem. Bull. Amer. Math. Soc. (N.S.), 39(3):301-354, 2002.

[15] Samuel Karlin and William J. Studden. Tchebycheff systems: With applications in analysis and statistics. Pure and Applied Mathematics, Vol. XV. Interscience Publishers John Wiley \& Sons, New York-London-Sydney, 1966.

[16] Jibin Li. Hilbert's 16th problem and bifurcations of planar polynomial vector fields. Internat. J. Bifur. Chaos Appl. Sci. Engrg., 13(1):47-106, 2003.

[17] Yongkun Li and Bing Li. Almost periodic time scales and almost periodic functions on time scales. J. Appl. Math., pages Art. ID 730672, 8, 2015.

[18] Alcides Lins Neto. On the number of solutions of the equation $d x / d t=\sum_{j=0}^{n} a_{j}(t) x^{j}, 0 \leq t \leq 1$, for which $x(0)=x(1)$. Invent. Math., 59(1):67-76, 1980.

[19] Noel G. Lloyd. The number of periodic solutions of the equation $\dot{z}=z^{N}+p_{1}(t) z^{N-1}+\ldots+p_{N}(t)$. Proc. London Math. Soc. (3), 27:667-700, 1973.

[20] Noel G. Lloyd. On a class of differential equations of Riccati type. J. London Math. Soc. (2), 10:1-10, 1975.

[21] Noel G. Lloyd. A note on the number of limit cycles in certain two-dimensional systems. J. London Math. Soc. (2), 20(2):277-286, 1979. 
[22] Viktor A. Pliss. Nonlocal problems of the theory of oscillations. Translated from the Russian by Scripta Technica, Inc. Translation edited by Harry Herman. Academic Press, New York-London, 1966.

[23] Sabrina H. Streipert. Abel dynamic equations of the first and second kind. Master's thesis, Missouri S\&T, 2012.

[24] Leonid A. Čerkas. The number of limit cycles of a certain second order autonomous system. Differencial' nye Uravnenija, 12(5):944-946, 960, 1976.

[25] Chao Wang and Ravi P. Agarwal. A further study of almost periodic time scales with some notes and applications. Abstr. Appl. Anal., pages Art. ID 267384, 11, 2014.

Department of Mathematics, Missouri S\&T, Rolla, Missouri 65409-0020, USA

E-mail address: bohner@mst.edu

Departament de Matemàtiques, Universitat Autònoma de Barcelona, 08193 Bellaterra, Barcelona, Catalonia, Spain

E-mail address: gasull@mat.uab.cat

Departamento de Matemática, Instituto Superior Técnico, Av. Rovisco Pais 1049-001, Lisboa, Portugal

E-mail address: cvalls@math.ist.utl.pt 